\title{
Nutritional Support for Critically Ill Patients at Home
}

\section{Leonidas Grigorakos ${ }^{1,2 *}$, Daria Lazarescu ${ }^{1}$, Magdalini Nikolaou ${ }^{1}$, Dimitra Markopoulou ${ }^{2}$, Athina Kalokairinou ${ }^{1}$ and Pavlos Myrianthefs ${ }^{1}$}

${ }^{1}$ Faculty of Nursing, National and Kapodistrian University of Athens, Greece

${ }^{2}$ Intensive Care, "KAT" Trauma Hospital of Athens, Kifissia

*Corresponding Author: Leonidas Grigorakos, Professor, National and Kapodistrian University of Athens, Greece.

Received: August 22, 2019; Published: September 06, 2019

\begin{abstract}
Over the past forty years laboratory studies and extensive epidemiological data evidenced that nutrition plays an important role in the quality of life, in the prevention, improvement and treatment of many pathological conditions. This is also the case for critically ill patients who need enteral and/or parenteral nutrition at home. This article aims to provide a multiple approach to critically ill patients with nutritional problems at home, combining clinical experience in Greece and international bibliographic data. It concludes that although enteral and parenteral nutrition at home represent a challenging area of research due to endless difficulties, government departments in charge of health should develop specific programs that raise the level of care of critically ill patients at home and offer them better life conditions are essential.

Keywords: Enteral Nutrition; Parenteral Nutrition; Critically Ill Patients; Nutritional Support
\end{abstract}

\section{Abbreviations}

EN: Enteral Nutrition; PN: Parenteral Nutrition.

\section{Introduction}

Major developments in medicine, chemistry and biochemistry relate to developments in the field of nutrition as well. Over the past forty years laboratory studies and extensive epidemiological data evidenced that nutrition plays an important role in the quality of life, in the prevention, improvement and treatment of many pathological conditions.

During the '80s developed countries gave emphasis to educating physicians, dieticians and nurses on the major issues of nutrition, with special departments and teams of specialized staff (doctors, dieticians, nurses) in all major hospitals. In Greece, even nowadays there are few doctors and nutritionists who are able to effectively apply optimum nutritional support to clinical practice. However, recent evidence shows that the collaboration of few but well-trained specialists is crucial for the benefit of patients and science [1]. Thus, major technological advances in nutritional support methods help nutritional needs of patients with enteral (EN) or parenteral nutrition (PN) to be met. These methods of nutritional support can be used by the patient himself, allowing him the independence and an increased sense of well-being. Patients requiring nutritional support at home, if their disease does not require continued clinical care or restrictions, may be full-time employed or travel.

When therapeutic acts and methods are used cautiously under appropriate indications, complications are minimized. The successful implementation of nutritional support at home depends on the harmonious co-operation of professionals dealing with the patient, aiming to solve medical problems, explain and apply appropriate guidance to the patient or his family, and address economic and social problems.

Patients may need nutritional support at home for a short period of time -such as during chemotherapy, irradiation, or other conditions that affect nutritional support at home -, because their illness is severe or if they have a permanent disability which leads to feeding inability. 
Currently, if patient' s general condition requires it, EN and PN methods used in the hospital can be easily used at home too [2].

This article aims to provide a multiple approach to critically ill patients with nutritional problems at home, combining clinical experience in Greece and international bibliographic data.

\section{The role of dietician in EN and PN at home}

The cooperation of medical and nursing staff with the hospital' s Nutrition Department is considered necessary since 1 out of 3 patients treated in pathological or surgical clinics are malnourished [3]. As the dietitian does not have time to monitor all patients in the hospital, experienced multidisciplinary teams that monitor patients whose nutrition is considered of primary importance are absolutely necessary. These teams should include a physician, a nurse and a dietician. Nursing staff is able to monitor the diet of patients while the good cooperation between dieticians and nursing staff is vital [4]. The role of the dietitian is therefore multifaceted and his involvement in the EN and PN of hospitalized patients and at home becomes an urgent need (Table 1).

1. Identify patients who need nutritional support at home.

2. Patient's monitoring.

3. Control of food and systems for use.

4. Patient's and family's education.

5. Checking the operation of food supply systems.

6. Rating: Success - Failure.

7. Research and development.

8. Social intervention: information about emerging problems psychological support for patients and relatives.

Table 1: The role of dietician in EN and PN.

\section{Nutrition assessment}

Nutrition assessment is part of patient's general medical examination. Nutrition assessment includes: historical and physical examination, anthropometric methods, biochemical methods, immunological parameters, nutrient balance study, dynometry (Table 2).

However, the ability of the above indicators to predict complications of malnutrition or to guide nutritional management is still questionable. In clinical practice, history and physical condition remain the cornerstone of patients' therapeutic condition. Nutrition status and dietary evaluation from the clinical dietologist should complement the medical history. Nutritional support at home should therefore be designed according to patients's historical and nutritional requirements (Table 3). Laboratory tests such as: albumin, vitamin levels, magnesium, phosphorus, potassium, zinc and iron are useful in assessing nutrition.

\section{Weight loss}

Anorexia

History of malabsorption

Weakness

Loss of muscle mass

Natural signs indicating a lack of specific coponents

(e.g. hypocalcaemia)

\section{Anthropometric methods}

Body weight

Thickness of skin

Arm muscle circumference

Arm muscle surface

\section{Biochemical methods}

Kreatinine height index

3 urine methylstidine $/ 24 \mathrm{~h}$

Serum Albums: Albumin, Prealbumin, Transferin

Minerals, vitamins, minerals

\section{Immunobiological parameters}

Reaction to skin tests

Total number of lymphocytes

Serum immunoglobulins

\section{Nutrient balance study}

Nitrogen balance

Balance of trace elements and minerals

Basic energy needs

\section{Dynometry}

Study of the effect of the patient's metabolic reaction on their pre-existing nutritional status.

Table 2: Patient's history and physical examination 
- $\quad$ Recent weight loss over $5 \%$

- Inability to get food

- Gastrointestinal malfunction as a result of chemotherapy

- Abdominal surgery

- $\quad$ Severe diarrhea

- Motor neuron diseases and

- Myopathies with respiratory failure

Table 3: Indications of nutritional support at home

\section{EN at home}

Oral fluid administration is necessary if the patient can swallow. EN is considered necessary when patients cannot be fed orally adequately but do have functional guts. This is the case of patients with: traumatic brain injury, coma, cervical cancer, esophageal tumors, short bowel syndrome, strokes, motor neuron disease, myopathies, anorexia. It is considered if: a) the nasal tube can be easily used for home nutrition, and b) the tube is inserted by the doctor and is kept for use at home.

Some patients or relatives quickly learn to change the nasal tubes and to control their position [5]. However, it should be emphasized that every patient who is eligible for EN at home must start it during the hospitalization period so that any metabolic or intolerance problems resulting from EN to be addressed.

The amount of EN should be gradually increased until the patient's nutritional needs are met. Because the volume of the administered food often causes problems, it may be necessary that the administered solution with higher concentration or frequency of administration to decrease according to the patient's tolerance.

The type of solution given will be decided jointly and with the agreement of the physician and the dietitian, as currently there are dozen types of nutrition formulations and specific solutions for each disease [6].

Patients who have fluid loss due to diarrhea or vomiting usually develop electrolytic disorders. These patients need to be monitored for sugar, sodium, potassium, magnesium and phosphorus and re-administration of any losses is necessary.

Attention must be paid both to the compatibility of the solution given with the medication and any obstruction of the tube. Liquid administration of the medication or trituration of the tablets is preferable to leaching of the nasal or other tube.
The instructions given to patients and their family members about EN at home should be clear and understandable. The type of solution, the frequency of administration, the volume to be administered on a daily basis, the volume increase per day, the method of administration, the liquids and methods required should be explained in detail. Also, the therapeutic goal, such as maintaining or increasing the weight of the solution after a certain period of time, must be understood by the patient and his/her environment. The patient should be examined by an expert team every 10 days. Nowadays, in the USA there are special programs for EN at home, covered by insurance companies. There are special committees certifying for 3, 8, or 18 months the need for EN at home.

Special home pumps are used with extreme caution and only if gravity or bolus administration cannot be used or is dangerous to the patient [7].

Great success for the US health care system is the existence of companies that supply enteral solutions and provide paramedical staff at home for EN and during hospitalization.

The combination of EN and PN at home is not uncommon because many patients cannot meet their nutritional needs only with enteral nutrition.

PN at home

The criteria for total PN at home include:

1. Stable clinical condition

2. Inability to get food in other ways

3. Perception and acceptance of the necessary administration of total PN

4. Good support system, presence and desire to help from family members

5. Desire to learn techniques and ability to apply them.

The physical condition of the patient prior to the establishment of PN should be monitored and the possible administration of the nutrition by the patient himself, which makes him independent and restores normal life and occupation, should be maximized by his integration into a rehabilitation and physiotherapy program of hospital where he was hospitalized.

The PN education program at home should be attended by all family members, even children, depending on their level of perception. 
The training is provided by demonstration, follow-up and written instructions. The way solutions are prepared, the use of syringes and needles, as well as all the devices and the catheter used, is well known by all involved in the patient's PN at home.

Contact with other patients receiving PN at home, sharing opinions and answering any questions are considered important steps before starting this type of diet.

The composition of the solutions is determined by the doctor and the dietician. Patients and relatives are informed of any complications such as hyperglycaemia, electrolyte abnormalities, dehydration, edema, and infection.

Patients are advised that the onset of hyperglycaemia may be a sign of sepsis. Emergencies and problems are discussed and analyzed during training. Frequent telephone contact with the physician, dietician or nurse is considered necessary, and any changes to the schedule are discussed during hospital or clinic visits. The slightest suspicion of sepsis is reported immediately to the treating physician. Emphasis is placed on aseptic solution preparation techniques and catheter care. All patients on PN should receive prophylactic treatment following dental or other interventions.

As the patient prepares to leave the hospital and go home, where he or she will continue PN, everything (e.g. the availability of solutions, pumps, sanitary equipment and agreement with the provider of the solution/ home infusion pharmacist) should be ready, so that the patient and the environment feel safe [8].

\section{Discussion and Conclusions}

Sometimes patients and/or their environment are reluctant to accept the necessity of either EN or PN. The majority of these patients have a negative attitude to their health and their dependence on EN or PN. Over time, however, they accept this diet as part of their lives and quickly learn how and what kind of diet they can get and tolerate [9].

Contact with the dietitian is invaluable and should be sought. The dietician can help with dietary adjustments and advise relatives that their dietary habits should not change because a family member cannot have lunch with them and should not feel guilty when eating with the patient. Pain management of some patients due to their general condition should be treated by a specialist.

The time of administration of the solutions should be adapted to the patient's lifestyle. Holidays and trips should be encouraged, provided that any complications or shortcomings can be addressed at the holiday location. Psychiatric follow-up of patients at predetermined intervals is considered necessary $[10,11]$.

As in the case of EN, so for PN nutrition at home, in the USA but also in the United Kingdom there are companies that supply and monitor patients under the supervision of an insurance provider. Dietitians and nurses visit patients daily, monitor the course of the disease, inform doctors and certify the state of health and care provided to critically ill patients.

Ongoing EN and PN programs that are in progress are intended to improve the living conditions of critically ill patients within a human-family environment.

Finally, although EN and PN at home represent a challenging area of research due to endless difficulties, government departments in charge of health should develop specific programs that raise the level of care of critically ill patients at home and offer them better life conditions are essential.

\section{Conflict of Interest}

Authors declare that they don't have any financial interest or any conflict of interest.

\section{Bibliography}

1. Bischoff SC., et al. "Organisation, regulations, preparation and logistics of parenteral nutrition in hospitals and homes the role of the nutrition support team - Guidelines on Parenteral Nutrition". GMS German Medical Science 7 (2009).

2. Hurt RT and Steiger E. "Early history of home parenteral nutrition: from hospital to home". Nutrition in Clinical Practice 33(2018): 598-613.

3. Sauer AC., et al. "Prevalence of Malnutrition Risk and the Impact of Nutrition Risk on Hospital Outcomes: Results From nutrition Day in the US". Journal of Parenteral and Enteral $\mathrm{Nu}$ trition (2019): 1-8.

4. Ojo O and Brooke J. "Recent Advances in Enteral Nutrition". Nutrients 8.11 (2016): 709.

5. Kovacevich D., et al. "American Society for Parenteral and Enteral Nutrition Guidelines for the Selection and Care of Central Venous Access Devices for Adult Home Parenteral Nutrition Administration". Journal of Parenteral and Enteral Nutrition 43.1 (2019): 15-31. 
6. Ross CA., et al. "Modern nutrition in health and disease. 11th edition. Philadelphia: Lippincott Williams and Wilkins (2014).

7. Bischoff S., et al. "ESPEN guideline on home enteral nutrition". Clinical Nutrition (2019).

8. Bloch A and Brown P. "Methods of Nutritional Support in the Home". Journal of Pain and Symptom Management 5 (1990): 297-306.

9. Tsang PY and Carey S. "Impact of home parenteral nutrition on daily life: A qualitative study of eight patients". Nutrition and Dietetics 72.1 (2013): 16-21.

10. Kumpf V. "Challenges and Obstacles of Long-Term Home Parenteral Nutrition". Nutrition in Clinical Practice 34 (2019): 196-203.

11. Faruquie SS., et al. "Evaluation of patient quality of life and satisfaction with home enteral feeding and oral nutrition support services: a cross-sectional study". Australian Health Review 40.6 (2016): 605-612.

Volume 3 Issue 10 October 2019

(C) All rights are reserved by Leonidas Grigorakos., et al. 